\title{
A novel technique for awake, minimally invasive transforaminal lumbar interbody fusion: technical note
}

\author{
Andrew Kai-Hong Chan, MD, Winward Choy, MD, Catherine A. Miller, MD, \\ Leslie C. Robinson, MD, PharmD, MBA, and Praveen V. Mummaneni, MD \\ Department of Neurological Surgery, University of California, San Francisco, California
}

\begin{abstract}
Minimally invasive transforaminal lumbar interbody fusion (MI-TLIF) is associated with improved patient-reported outcomes in well-selected patients. Recently, some neurosurgeons have aimed to further improve outcomes by utilizing multimodal methods to avoid the use of general anesthesia. Here, the authors report on the use of a novel awake technique for MI-TLIF in two patients. They describe the successful use of liposomal bupivacaine in combination with a spinal anesthetic to allow for operative analgesia.
\end{abstract}

https://thejns.org/doi/abs/10.3171/2019.1.FOCUS18510

KEYWORDS awake; minimally invasive; transforaminal lumbar interbody fusion; TLIF; spine; anesthesia

$\mathrm{M}$ INIMALLY invasive (MI) techniques have long been advocated as a means to decrease blood loss, shorten hospitalization, and expedite postoperative recovery. These techniques have been applied to transforaminal lumbar interbody fusion (MI-TLIF) with promising results in well-selected patients. In an effort to further improve outcomes following MI-TLIF, some neurosurgeons have advocated the use of multimodal methods that avoid the use of general anesthesia.

Wang and Grossman first reported the efficacy and safety of an approach utilizing a combination of endoscopic techniques, expandable bone grafts, percutaneous fixation, osteobiologics, and a long-acting local anesthetic to achieve the goal of surgery sans general anesthesia. ${ }^{13}$ In the first 10 consecutive patients who underwent their endoscopic MI-TLIF with a minimum 12-month follow-up, the authors noted a mean operative time of $113.5 \pm 6.3$ minutes and a mean hospitalization of $1.4 \pm 1.3$ nights without any intraoperative or postoperative complications. On postoperative radiographic follow-up (flexion-extension radiographs), there were no cases of nonunion at 12 months. In terms of patient-reported metrics, patients improved significantly in both disability and health-related quality of life, as measured by the EQ-5D. Notably, none of the patients required conversion to general anesthesia or open surgery.

The technique reported by Wang and Grossman and the awake, fully endoscopic MI-TLIF described by Telf- eian are notable efforts in enhancing recovery following MI-TLIF. ${ }^{10,13}$ However, there are several drawbacks. First, the reliance on endoscopic decompression is limiting and may not be appropriate for patients with severe central or bilateral foraminal stenosis. Second, Wang and Grossman noted a 120-minute surgical cutoff time for their procedure, which may limit its broad application. Additionally, it is unclear if there is generalizability of such a procedure across institutions and anesthesia-surgical teams.

Here, we report on the successful use of a novel technique for MI-TLIF that helps to address the above concerns. We rely on a nonendoscopic direct-decompression technique performed through tubular retractors, allowing for direct microscopic decompression. We use a spinal anesthetic in order to improve pain outcomes and allow surgery to extend beyond 120 minutes. We report on the safety of using liposomal long-acting local anesthetics and show that methods to conduct MI-TLIF without general anesthesia are reproducible. ${ }^{10,13}$

\section{Operative Technique}

Perioperative antibiotics are administered in the usual fashion. The patient first receives a spinal anesthetic. We do not utilize neurophysiological monitoring (e.g., electromyography). The anesthetic protocol is outlined in Table 1. Patients position themselves prone on a Jackson Table

ABBREVIATIONS EBL = estimated blood loss; IONM = intraoperative neuromonitoring; JP = Jackson-Pratt; MI = minimally invasive; TID = three times a day; TLIF = transforaminal lumbar interbody fusion; VAS = visual analog scale.

SUBMITTED September 27, 2018. ACCEPTED January 15, 2019.

INCLUDE WHEN CITING DOI: 10.3171/2019.1.FOCUS18510. 
TABLE 1. Perioperative protocol for awake MI-TLIF

\begin{tabular}{|c|c|}
\hline Management & Description \\
\hline Preop medication & Acetaminophen 1000 mg; gabapentin 600 mg (hold for CKD w/ GFR <60, age >70 yrs) \\
\hline \multicolumn{2}{|l|}{ Intraop management } \\
\hline Preprocedure sedation & Midazolam per anesthesiologist (ideally <2 mg); fentanyl per anesthesiologist (ideally <100 ㅆg) \\
\hline Lumbar spinal & $\begin{array}{l}15 \mathrm{mg} \text { of isobaric bupivacaine ( } 3 \text { cc } 0.5 \% \text { bupivacaine, preservative free); } 10-25 \mu \mathrm{g} \text { fentanyl; injected } 1 \text { space } \\
\text { above or below operated level }\end{array}$ \\
\hline Sedation & $\begin{array}{l}\text { Propofol titrated to Ramsay Sedation Scale score of } 2-3(25-50 \mu \mathrm{g} / \mathrm{kg} / \mathrm{min}) \text {; ketamine } 2 \mu \mathrm{g} / \mathrm{kg} / \mathrm{min} \text {; limit opi- } \\
\text { ates; alternate options: dexmedetomidine gtt, fentanyl/midazolam }\end{array}$ \\
\hline Blood pressure support & Consider fluid bolus; phenylephrine gtt vs ephedrine to maintain MAP $65 \mathrm{~mm} \mathrm{Hg}$ or $80 \%$ baseline \\
\hline Nausea prophylaxis & Dexamethasone $4 \mathrm{mg} \mathrm{IV} \times 1$ (hold for patients w/ diabetes mellitus); Zofran $4 \mathrm{mg} \mathrm{IV} \times 1$ \\
\hline Surgical infiltration & $\begin{array}{l}\text { Infiltration of } 10 \mathrm{ml} \text { of liposomal bupivacaine ( } 20 \mathrm{ml} \text { of } 1.3 \% \text { liposomal bupivacaine diluted } \mathrm{w} / 20 \mathrm{ml} \text { of normal } \\
\text { saline to total vol of } 40 \mathrm{ml} \text { ) to each incision/percutaneous screw tract }\end{array}$ \\
\hline For inadequate analgesia after $2 \mathrm{hrs}$ & Re-inject $1 \mathrm{ml}$ of $0.5 \%$ bupivacaine $w / 24$-gauge pencil-tip spinal needle on surgical field \\
\hline \multicolumn{2}{|l|}{ Postop management } \\
\hline Pain control & $\begin{array}{l}\text { Avoid PCA; acetaminophen } 1000 \mathrm{mg} \text { q4h ATC; gabapentin } 300 \mathrm{mg} \text { PO TID; oxycodone } 5 \mathrm{mg} \text { PO q3h PRN } \\
\text { for moderate pain; hydromorphone } 0.2-0.4 \mathrm{mg} \text { IV q3h PRN for severe pain unresponsive to PO analgesics } \\
\text { (avoid if possible) }\end{array}$ \\
\hline Nausea control & Zofran 4 mg IV q6h PRN for nausea and/or vomiting \\
\hline Voiding & Check bladder scan if unable to urinate in PACU, straight catheter PRN \\
\hline Mobilization & Physical therapy day of surgery \\
\hline Disposition & w/in 24 hrs \\
\hline
\end{tabular}

ATC = around the clock; $C K D=$ chronic kidney disease; GFR = glomerular filtration rate; gtt = guttae (drops); IV = intravenous; MAP = mean arterial pressure; PACU = postanesthesia care unit; PCA = patient-controlled analgesia; $P O=$ per os (by mouth); $P R N=$ when necessary; q3h, .., q6h = every 3 hours, . ., every 6 hours; $T I D=$ three times a day.

while awake. We prepare the patient in the usual fashion and drape them sterilely. First, attention is placed on the iliac crest. Iliac bone marrow autograft aspirate is harvested with a Jamshidi needle and is augmented with graft extender. Next, we place the reference frame of the Surgical Navigation System (Medtronic Inc.) on the right iliac crest. We then obtain a navigated CT scan using a portable scanner (Fig. 1A-C). We then register and confirm landmarks for spinal navigation.

Two 1-inch incisions are planned in the Wiltse plane utilizing navigation (Fig. 1E). Then, prior to skin incision, an injectable liposomal bupivacaine suspension (Exparel, Pacira Pharmaceuticals Inc.) is infiltrated into both incisions and screw trajectories (Fig. 1B and E). This provides approximately 72 hours of local analgesia.

Next, the L4-5 pedicle screws are placed using navigation (Fig. 1D). First, a navigated screw with a cutting flute is placed into the cranial pedicle (Viper Prime, DePuy Synthes). We leave the caudal screw (i.e., L5) out ipsilateral to the side of interbody placement until after placement to facilitate visualization. Next, a contralateral partial facetectomy is completed. The bone is kept as local autograft. Subsequently, a drill is utilized to decorticate the contralateral L4-5 facet. The local autograft is placed on the decorticated contralateral L4-5 facet for a posterolateral fusion.

Next, the ipsilateral facet is removed with a combination of a drill and Kerrison rongeur. Again, the bone is kept as local autograft. The exiting nerve root can be further decompressed using an operative microscope.

Next, a discectomy is completed through the tubular re- tractor. A combination of shavers and curettes are utilized to prepare the disc space. Subsequently, interbody trials of sequentially larger sizes are placed until an appropriate fit is visualized (Fig. 1F). Next, the TLIF interbody expandable cage, filled with local autograft, is placed. We prefer to augment our interbody placement with iliac crest bone marrow aspirate and local crest autograft and graft extender.

After interbody cage placement, the remaining caudal ipsilateral pedicle screw is inserted. Rods are placed and locked down into the construct. Hemostasis is achieved with bipolar cautery. The wound is then closed in a layered fashion. Anteroposterior and lateral intraoperative fluoroscopy is utilized to confirm optimal location of the hardware (Fig. $1 \mathrm{G}$ and $\mathrm{H}$ ). A video of the technique is provided (Video 1).

VIDEO 1. Video depicting the awake, minimally invasive transforaminal lumbar interbody fusion. Copyright Andrew Chan. Published with permission. Click here to view.

\section{Illustrative Cases \\ Case 1}

History and Examination

This 63-year-old man had scoliosis and Meyerding grade I L4-5 spondylolisthesis with low-back pain and left leg radiculopathy, including parasthesias (Fig. 2A and B). His imaging revealed a lateral L4-5 disc herniation, scoliosis, advanced degeneration, and left-sided up/down foraminal stenosis (Fig. 2E and F). He had received a trial 
of oral prednisone, naproxen $500 \mathrm{mg}$ as necessary, gabapentin, lumbar physical therapy, and four lumbar epidural steroid injections without significant relief. He refused to undergo a fusion procedure, so he underwent a left L4-5 MI laminotomy and foraminotomy but continued to have radicular pain.

His postoperative course was notable for a recurrence of his leg pain several weeks after surgery despite initial symptom relief. Postoperative MRI revealed persistent left L4-5 up/down foraminal stenosis compressing the exiting L4 nerve root (Fig. 2G). There was no recurrent disc herniation.

\section{MI-TLIF}

Two months after his initial surgery, he underwent an awake L4-5 MI-TLIF with neuronavigation. A left-sided facetectomy and asymmetrical cage placement to the left was completed to correct the up/down foraminal stenosis (Fig. 2H). A Jackson-Pratt (JP) drain was placed to suction. Estimated blood loss (EBL) was under $100 \mathrm{ml}$. The operative time was 2.5 hours. There were no perioperative complications.

\section{Postoperative Course}

The patient mobilized within 4 hours of surgery and was able to ambulate 300 feet with an assistive device and to walk on stairs with physical therapy. Immediate postoperative radiographs demonstrated the hardware in a good position (Fig. 2C and D). His pain was well controlled. He required acetaminophen $1000 \mathrm{mg}$ three times a day (TID), baclofen $10 \mathrm{mg}$ TID, pregabalin $75 \mathrm{mg}$ TID (a home medication), and six oxycodone $10 \mathrm{mg}$ when necessary during his hospitalization. There were no requirements for any intravenous opioid or anti-nausea medications. The drain was removed on postoperative day 1 . The patient was tolerating a regular diet. The Foley catheter was removed, and the patient voided successfully. He was discharged to home without event on postoperative day 1 with the recommendation for outpatient physical therapy. He was discharged with oral oxycodone-acetaminophen 5-325 mg to be taken as needed for pain. He was given baclofen $10 \mathrm{mg}$ TID and a fentanyl patch as well.

At the 3-month postoperative follow-up, the patient noted $70 \%$ improvement in his sensory disturbance with only occasional burning pain on the medial dorsal aspect of his left foot at night when lying down. His pain was well controlled on acetaminophen alone and without narcotics. His visual analog scale (VAS) back and leg pain score was improved from 6 to 1 . He returned to work full-time.

\section{Case 2}

History and Examination

This 67-year-old female with scoliosis, Meyerding grade I L4-5 spondylolisthesis, and moderate lumbar stenosis presented with left leg pain radiating from her buttocks to her calf that had lasted for 1 year (Fig. 3A, B, E, and F). She also noted intermittent left foot parasthesias and a partial right foot drop. She had mild low-back pain but ambulated without difficulty. Preoperative dynamic lumbar radiographs revealed dynamic listhesis at L4-5

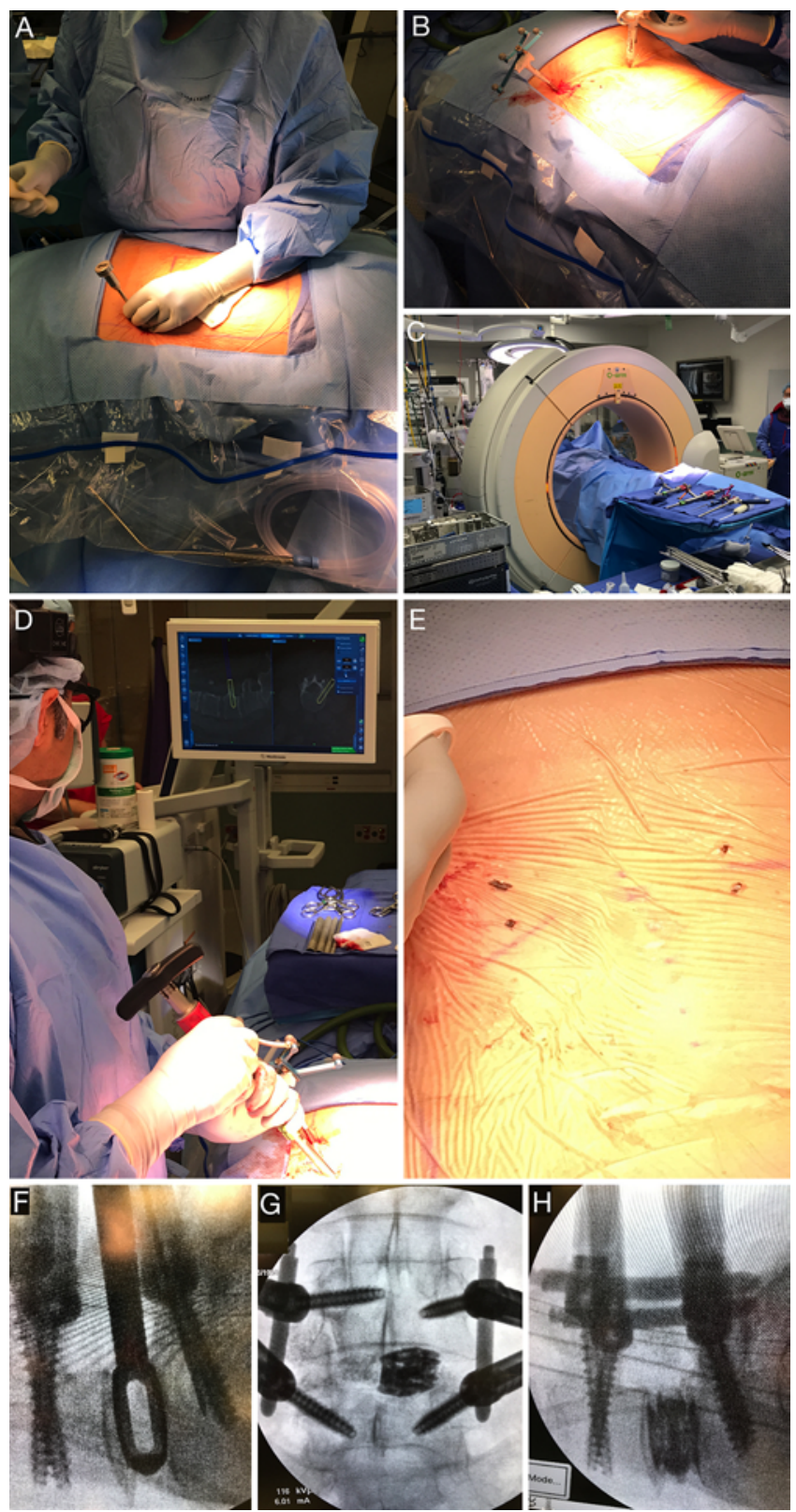

FIG. 1. A: The reference frame of the StealthStation Surgical Navigation System (Medtronic Inc.) is placed on the right iliac crest. B: The frame is placed inferolaterally, facilitating operative freedom while avoiding unintended displacement of the frame. C: A Stealth-protocol navigated CT scan is obtained with an 0 -arm. An additional sterile drape is placed over the operative field prior to closure of the 0 -arm, which is removed after the $\mathrm{O}$-arm spin is completed. $\mathrm{D}$ : The trajectory of the L4 MI cutting-flute percutaneous pedicle screw (Viper Prime, DePuy Synthes) is planned utilizing spinal navigation. E: Two 1-inch incisions are planned for an L4-5 TLIF. Liposomal bupivacaine (Exparel, Pacira Pharmaceuticals Inc.) is injected into the planned incisions and into the planned screw trajectories. F: Interbody trials of sequentially larger sizes are placed until an appropriate fit is visualized. $\mathbf{G}$ and $\mathrm{H}$ : Anteroposterior (AP) and lateral fluoroscopic images are obtained to confirm accurate interbody cage (Concorde Lift, DePuy Synthes) placement. 


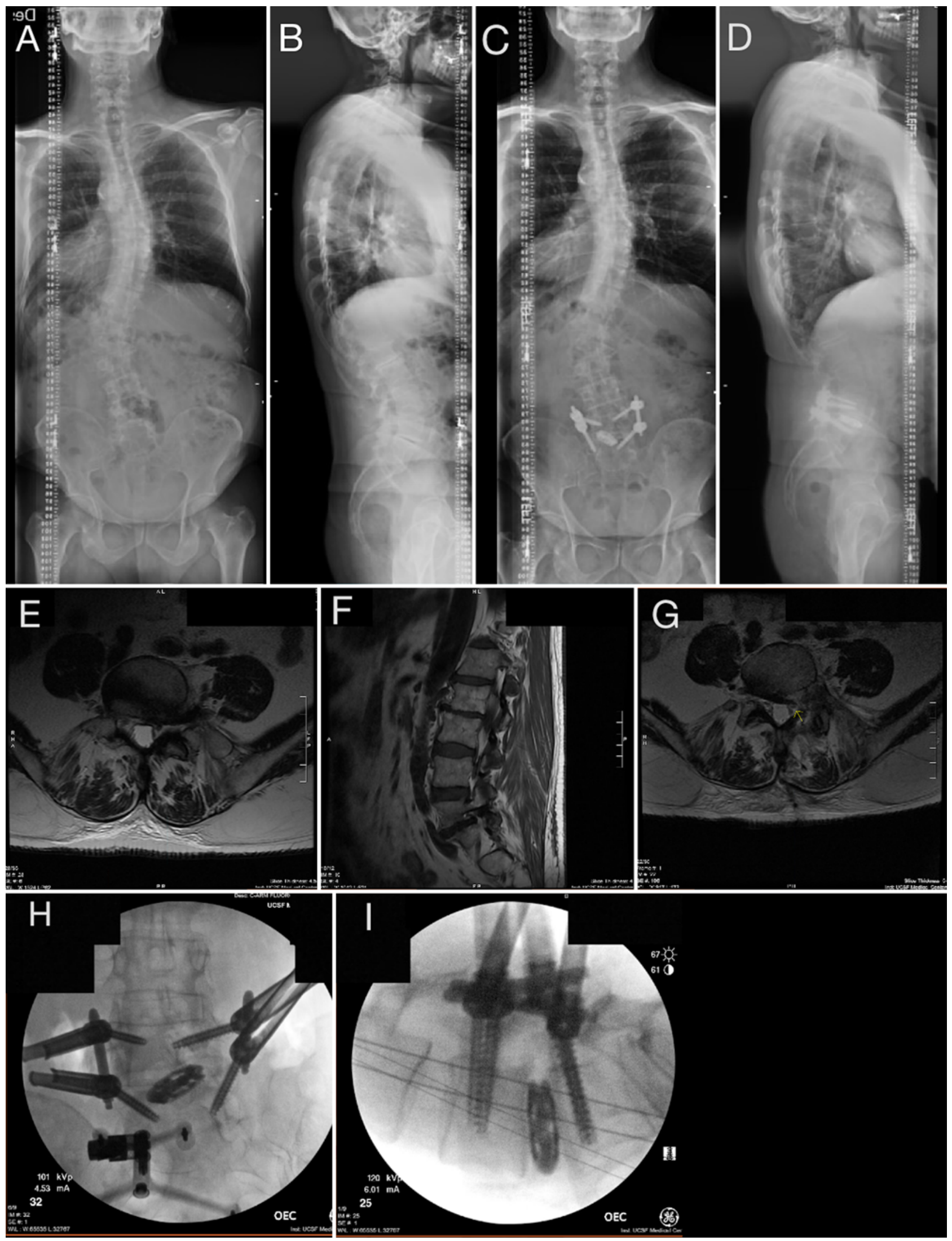

FIG. 2. Case 1. Preoperative AP (A) and lateral (B) radiographs demonstrating Meyerding grade I L4-5 spondylolisthesis and scoliosis. Postoperative AP (C) and lateral (D) radiographs obtained after L4-5 MI-TLIF, demonstrating hardware in the expected position. FIG. 2. (continued) $\rightarrow$ 
FIG. 2. Preoperative axial T2-weighted (E) and parasagittal T1-weighted (F) MR images reveal a lateral L4-5 disc herniation. The parasagittal T1-weighted MR image reveals left-sided foraminal stenosis. A postoperative axial T2-weighted MR image (G) obtained after MI decompression surgery, revealing persistent left $L 4-5$ foraminal stenosis compressing the exiting $L 4$ nerve root (arrow). Intraoperative AP $(\mathbf{H})$ and lateral (I) fluoroscopic images demonstrate hardware in a good position and asymmetrical cage placement to the left to correct the up/down foraminal stenosis.

(Fig. $3 \mathrm{G}$ and $\mathrm{H}$ ). The pain was resistant to naproxen 500 mg daily, massage therapy, physical therapy, and an epidural steroid injection. She also tried hydrocodone-acetaminophen, which did not have a significant effect.

\section{MI-TLIF}

The patient underwent an awake L4-5 MI-TLIF with percutaneous screw fixation and interbody cage placement from the left with the aid of neuronavigation (Fig. 3I and J). A JP drain was placed to suction. The EBL was under $100 \mathrm{ml}$. The operative time was 2.5 hours. There were no perioperative complications.

\section{Postoperative Course}

She mobilized immediately postoperatively with physical therapy. On postoperative day 1 , she was able to ambulate. Her JP drain was removed. She was discharged to home on postoperative day 1 . She was discharged with baclofen $5 \mathrm{mg}$ every 6 hours, gabapentin $300 \mathrm{mg}$ TID, and oxycodone $7.5 \mathrm{mg}$ to be taken every 4 hours.

At the routine 3-month follow-up, the patient noted some right lateral hip pain and low-back pain. Her radiographs revealed postsurgical findings (Fig. 3C and D). Her leg pain was much improved, and she had reduced her oxycodone use to once nightly. Her VAS back pain score worsened from 0 to 6 postoperatively. Her VAS leg pain score improved from 7.5 to 0 postoperatively.

\section{Discussion}

The present study demonstrated that MI-TLIF is well tolerated in the awake patient under conscious sedation. We also showed the feasibility of 1) a nonendoscopic technique for MI-TLIF performed through tubular retractors and 2) the combination of the off-label use of liposomal long-acting local anesthetics and a spinal anesthetic to allow for longer operative times ( $>2$ hours).

The goals of MI spine surgery include the decrease of complications, recovery times, postoperative pain, and costs. In alignment with this aim, the use of local anesthesia and conscious sedation in MI-TLIF offers a number of advantages. The risks associated with intubation and the induction of general anesthesia are eliminated, and adverse effects from this process, such as nausea and memory loss, can be avoided..$^{13}$ During recovery from general anesthesia, patients may have mental confusion and transient impairment of sensory and motor function. ${ }^{12} \mathrm{He}-$ modynamic instability and cardiac dysrhythmias can also occur during emergence from general anesthesia. Consequently, general anesthesia may be contraindicated in select patients, particularly in the elderly and those with a high perioperative risk due to medical comorbidities.

Authors reporting their experience with spine surgeries in awake patients have cited improved postoperative analgesia, shorter recovery times, decreased costs, and improved perioperative morbidity and mortality. ${ }^{6}$ Wang et al. reported decreased operative time, postoperative analgesic utilization, and costs in patients undergoing singlelevel anterior cervical discectomy and fusion with cervical plexus anesthesia as compared to those with general anesthesia. ${ }^{12}$ Conscious sedation allows the patient to remain awake and interact with the surgeon during critical steps that may involve the manipulation of nerve roots resulting in neurological symptoms. This immediate and live feedback can inform the surgeon of the patient's neurological status during positioning and the surgery itself and obviate the need for intraoperative neuromonitoring (IONM) as in this report. Decreased intrathoracic pressure from spontaneous - as opposed to mechanical-breathing likely reduces bleeding during the procedures. ${ }^{7}$ Calderon et al. similarly noted that the avoidance of general anesthesia in carotid endarterectomy has the benefit of intraoperative evaluation of the patient's neurological function, a shorter length of stay, and decreased rates of cerebrovascular accidents. $^{2}$

Although the use of a local anesthetic in MI spine surgery has been particularly described in the setting of decompression (e.g., discectomies or laminectomies), ${ }^{1,3,8,9,11,14}$ the literature on its application for MI-TLIF is limited. In a recent case report by Telfeian, a 42-year-old male with lumbar radiculopathy underwent awake L4-S1 MI-TLIF with the use of an endoscopic system, local anesthesia, and conscious sedation. ${ }^{10}$ The postoperative course was uncomplicated, and the patient noted the resolution of symptoms at the 1-year follow-up. Of note, the technique described by Telfeian relies on a side shaver drill for decompression that is fully reliant on fluoroscopy for visualization. This disadvantage, as the author notes, limits the ability to achieve a larger decompression, such as would be possible under direct visualization. With our technique, direct microscopic visualization is accomplished via the tubular retractor system, facilitating a wider decompression when necessary. Additionally, this nonendoscopic approach avoids the learning curve and expense associated with endoscopic equipment.

In the largest case series to date, Wang and Grossman reported on their experience with endoscopic single-level MI-TLIF in 10 patients without general anesthesia. ${ }^{13} \mathrm{~Pa}-$ tients were sedated with a ketamine and propofol infusion without the use of spinal, epidural, or regional anesthesia. The authors relied on an endoscopic technique for decompression. As in our series, the authors used off-label liposomal bupivacaine to provide long-acting local anesthesia. No case required conversion to open surgery. At the 1-year follow-up, the authors reported no postoperative complications, improvements in a number of health-related quality of life scores, and radiographic fusion on dynamic plain radiographs.

In contrast to their series, the present study utilized a nonendoscopic technique performed via tubular retrac- 

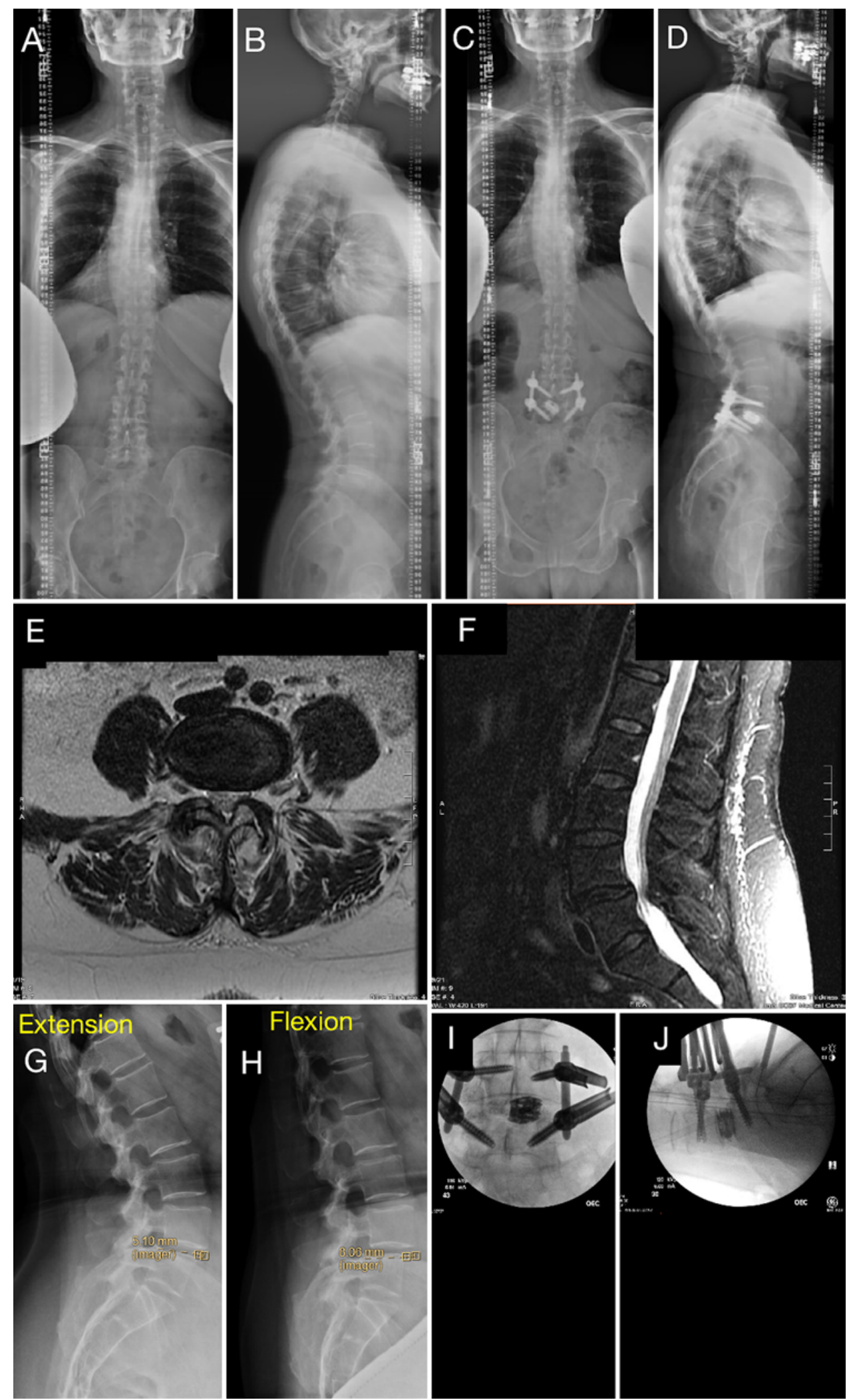

FIG. 3. Case 2. Preoperative AP (A) and lateral (B) radiographs demonstrating Meyerding grade I L4-5 spondylolisthesis and scoliosis. Postoperative AP (C) and lateral (D) radiographs demonstrating hardware in the expected position. Preoperative axial (E) and sagittal $(\mathbf{F})$ T2-weighted MR images reveal L4-5 lumbar stenosis. Dynamic extension (G) and flexion (H) lumbar plain radiographs demonstrate mobile spondylolisthesis. Intraoperative AP (I) and lateral (J) fluoroscopic images demonstrate the hardware in a good position. 
tors, allowing for a larger decompression and more thorough endplate preparation. Though this must be balanced with the greater soft tissue trauma, we believe this nonendoscopic method optimizes surgical decompression and augments fusion rates.

Wang and Grossman had a surgical cutoff time of 120 minutes, which may not be achievable by all surgical teams. However, the use of a spinal anesthetic in the present study, which was not used by Wang and Grossman, enhances perioperative pain control and thus allows surgery to extend well beyond 120 minutes (e.g., 2.5 hours in our cases). Given the longer surgical times permitted by the spinal anesthetic, more extensive, multilevel MI-TLIF may be possible, but further study is required. The disadvantage of the use of the spinal anesthetic in our technique is the inability to assess for complaints of pain, which would otherwise be helpful to monitor for compression of neural structures. These differences are aimed at augmenting the applicability and generalizability of awake MI-TLIF across institutions. While there are differences in surgical technique, the cumulative results demonstrate the feasibility of MI-TLIF in an awake patient. Importantly, the technique was well tolerated and safe, as there were no instances of nerve root, spinal cord, or dural injuries and no local tissue reaction to the injectable liposomal bupivacaine.

Of note, our technique avoids the use of IONM to minimize patient discomfort and decrease in-room operative time. Though IONM may aid in the identification of neurological injury during decompression, screw insertion, or interbody placement, ${ }^{4}$ the benefit in decreasing neurological injury, especially for smaller procedures like MI 1- and 2 -level transforaminal interbody fusion, ${ }^{5}$ has not been observed.

Despite its potential advantages, the procedure is not appropriate for all surgical candidates. Given the limitations on the duration of operative time, the procedure is best suited to patients who require surgery for 1- or 2-level lumbar stenosis or spondylolisthesis. The procedure is utilized for Meyerding grade I or II spondylolisthesis because these cases are conducted without IONM. We avoid the procedure in grade III or higher spondylolisthesis because we prefer to utilize IONM in such cases. Close airway monitoring is required for the procedure. Comorbid factors that compromise the airway, such as extreme obesity and obstructive sleep apnea, are relative contraindications to awake TLIF given that the airway is not secured during surgery. Patients with comorbid anxiety may not tolerate the conscious sedation required for the procedure. While pain can be well controlled with the techniques described, patients may feel uncomfortable and anxious during the surgery because of the long periods of prone positioning and the sounds of surgical instruments. This may additionally result in elevated heart rate or blood pressure. Given these considerations, select patients may benefit from additional propofol.

A control group in future studies will allow for an assessment of differences in recovery times from anesthesia, perioperative features, patient outcomes, and resource utilization based on the anesthetic approach, such as was undertaken by Wang et al. in their report of reduced costs following awake MI-TLIF as compared to conventional MI-TLIF. ${ }^{12}$ Further, long-term follow-up of the present cases, which is not within the scope of the present paper, will be important to clarify the efficacy of the procedure (e.g., radiographic fusion rates, long-term change in patient-reported outcomes, etc.).

\section{Conclusions}

Our preliminary experience demonstrates that a novel awake, tubular retractor technique using a microscope for MI-TLIF is feasible in patients who undergo spinal anesthesia. The advantages of our technique include the ability to achieve a wider decompression under direct visualization through tubular retractors and to operate for longer periods using a combination of liposomal bupivacaine and spinal anesthesia. Future prospective study with larger cohorts will be critical in fully defining the long-term efficacy of this technique.

\section{References}

1. Ames WA, Songhurst L, Gullan RW: Local anaesthesia for laminectomy surgery. Br J Neurosurg 13:598-600, 1999

2. Calderon AL, Zetlaoui P, Benatir F, Davidson J, Desebbe $\mathrm{O}$, Rahali N, et al: Ultrasound-guided intermediate cervical plexus block for carotid endarterectomy using a new anterior approach: a two-centre prospective observational study. Anaesthesia 70:445-451, 2015

3. Chopko BW: Percutaneous thoracolumbar decompression combined with percutaneous pedicle screw fixation and fusion: a method for treating spinal degenerative pain in a biplane angiography suite with the avoidance of general anesthesia. J Spine Surg 2:122-127, 2016

4. Fehlings MG, Brodke DS, Norvell DC, Dettori JR: The evidence for intraoperative neurophysiological monitoring in spine surgery: does it make a difference? Spine (Phila Pa 1976) 35 (9 Suppl):S37-S46, 2010

5. Garces J, Berry JF, Valle-Giler EP, Sulaiman WA: Intraoperative neurophysiological monitoring for minimally invasive 1- and 2-level transforaminal lumbar interbody fusion: does it improve patient outcome? Ochsner J 14:57-61, 2014

6. Griffin J, Nicholls B: Ultrasound in regional anaesthesia. Anaesthesia 65 (Suppl 1):1-12, 2010

7. Jellish WS, Thalji Z, Stevenson K, Shea J: A prospective randomized study comparing short- and intermediate-term perioperative outcome variables after spinal or general anesthesia for lumbar disk and laminectomy surgery. Anesth Analg 83:559-564, 1996

8. Sairyo K, Chikawa T, Nagamachi A: State-of-the-art transforaminal percutaneous endoscopic lumbar surgery under local anesthesia: discectomy, foraminoplasty, and ventral facetectomy. J Orthop Sci 23:229-236, 2018

9. Shtaya A, Luong CB, Pereira E: Awake intradural spinal tumor resection; case report and literature review. World Neurosurg 114:344-347, 2018

10. Telfeian AE: An awake, minimally-invasive, fully-endoscopic surgical technique for treating lumbar radiculopathy secondary to heterotopic foraminal bone formation after a minimally invasive transforaminal lumbar interbody fusion with BMP: technical note. J Spine Surg 4:162-166, 2018

11. Wan Q, Zhang D, Li S, Liu W, Wu X, Ji Z, et al: Posterior percutaneous full-endoscopic cervical discectomy under local anesthesia for cervical radiculopathy due to soft-disc herniation: a preliminary clinical study. J Neurosurg Spine 29:351-357, 2018

12. Wang H, Ma L, Yang D, Wang T, Wang Q, Zhang L, et al: 
Cervical plexus anesthesia versus general anesthesia for anterior cervical discectomy and fusion surgery: a randomized clinical trial. Medicine (Baltimore) 96:e6119, 2017

13. Wang MY, Grossman J: Endoscopic minimally invasive transforaminal interbody fusion without general anesthesia: initial clinical experience with 1-year follow-up. Neurosurg Focus 40(2):E13, 2016

14. Wu ZY, Zhu YJ, Chu L, Cheng CY, Chen CM, Hui-Ting H: Full-endoscopic transforaminal approach for removal of a spontaneous spinal epidural hematoma. World Neurosurg 98:883.e13-883.e20, e820, 2017

\section{Disclosures}

Dr. Mummaneni has served as a consultant for DePuy Spine, Globus, and Stryker; has received honoraria from AOSpine and Spineart; has direct stock ownership in Spinicity/ISD; has received royalties from DePuy Spine, Thieme Publishers, and Springer Publishing; and has received support from ISSG and NREF for non-study-related clinical or research effort. Dr. Chan has received support from Orthofix for non-study-related research.

\section{Author Contributions}

Conception and design: Chan, Mummaneni. Acquisition of data: Chan, Miller, Robinson, Mummaneni. Analysis and interpretation of data: Chan, Miller, Mummaneni. Drafting the article: Chan, Choy, Mummaneni. Critically revising the article: all authors. Reviewed submitted version of manuscript: all authors. Approved the final version of the manuscript on behalf of all authors: Chan. Study supervision: Mummaneni.

\section{Supplemental Information \\ Videos}

Video 1. https://vimeo.com/316084999.

\section{Correspondence}

Andrew K. Chan: University of California, San Francisco, CA. andrew.chan@ucsf.edu. 